\section{A E E T}

ASOCIACIÓN ESPAÑOLA DE ECOLOGÍA TERRESTRE

\section{ecosistemas}

REVISTA CIENTÍFICA DE ECOLOGÍAY MEDIO AMBIENTE

\title{
La vulnerabilidad de las especies frente al cambio climático, un reto urgente para la conservación de la biodiversidad
}

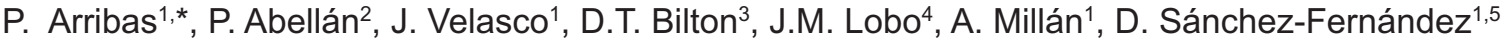

(1) Departamento. Ecología e Hidrología. Universidad de Murcia. 30100. Murcia. España.

(2) Department of Biological Sciences. Aarhus University. DK-8000. Aarhus. Dinamarca.

(3) Marine Biology and Ecology Research Centre. University of Plymouth. PL4 8AA. Plymouth. Reino Unido.

(4) Dpto. Biodiversidad y Biología Evolutiva. Museo Nacional de Ciencias Naturales (CSIC). 28006. Madrid.

(5) Instituto de Biología Evolutiva (CSIC-UPF). 08003. Barcelona.

*Autor de correspondencia: P. Arribas [pauarribas@um.es]

> Recibido el 9 de julio de 2012, aceptado el 17 de agosto de 2012.

Arribas, P., Abellán, P., Velasco, J., Bilton, D.T., Lobo, J.M., Millán, A., Sánchez-Fernández, D. (2012). La vulnerabilidad de las especies frente al cambio climático, un reto urgente para la conservación de la biodiversidad. Ecosistemas 21(3):79-84. Doi.: 10.7818/ECOS.2012.21-3.10 El actual cambio climático constituye una de las principales amenazas para el futuro de la biodiversidad. A pesar de que recientemente se han realizado numerosos estudios evaluando los efectos del cambio climático sobre la biodiversidad, esta información apenas se ha traducido en propuestas de gestión concretas que permitan minimizar su impacto sobre las especies. En este trabajo se recogen algunos de estos estudios (con especial énfasis en los centrados en la Península lbérica), y se discuten (con ejemplos) algunos conceptos fundamentales en este campo, como los factores que determinan la vulnerabilidad de una especie frente al cambio climático y su vinculación con propuestas de estrategias de gestión concretas.

Palabras clave: calentamiento global, adaptación al cambio climático, especies amenazadas, espacios protegidos, respuestas de las especies, estrategias de gestión

Arribas, P., Abellán, P., Velasco, J., Bilton, D.T., Lobo, J.M., Millán, A., Sánchez-Fernández, D. (2012). Species vulnerability under climate change, a new challenge for biodiversity conservation. Ecosistemas 21(3):79-84. Doi.: 10.7818/ECOS.2012.21-3.10

The current climate change is one of the main threats for the future of the biodiversity. Despite many recent studies have assessed the effects of the ongoing climate change on biodiversity, this information has not been translated into specific management strategies to minimize its impact on species. Here we summarize some of these studies (especially those focused on the lberian Peninsula) and discuss (including examples) about some fundamental concepts in this field, such as the factors determining species vulnerability under climate change and their potentialities to make concise proposals on conservation strategies.

Key words: climate warming, climate change adaptation, threatened species, protected areas, species responses, management strategies

El cambio climático al que nos enfrentamos en la actualidad constituye una de las principales amenazas para el futuro de la biodiversidad (Sala et al. 2000; Parmesan 2006), especialmente en regiones como la Cuenca Mediterránea (ver Cuadro 1 para una breve descripción de los cambios estimados en las condiciones climáticas de la Península Ibérica). Dicha amenaza plantea nuevos desafíos a la biología de la conservación, como la adaptación de sus herramientas y principios clásicos para incorporar las diferentes respuestas de los organismos ante el cambio climático, que es fundamental para llevar a cabo una gestión efectiva de las especies y sus hábitats (Akcakaya et al. 2006; Thomas et al. 2011).

Aunque en la actualidad existen numerosos estudios científicos que han tratado de ofrecer aproximaciones para evaluar las respuestas potenciales de distintos grupos de organismos ante el calentamiento global (ej. Thomas et al. 2004; Thuiller et al. 2005, 2011; Ohlemuller et al. 2006), esta información apenas se ha traducido en propuestas concretas de gestión que permitan minimizar los impactos del cambio climático sobre las especies y los sistemas naturales (Kareiva et al. 2008; Heller y Zavaleta 2009). Este hecho deja patente que, a pesar de que las estrategias para la conservación de la biodiversidad necesitan basarse en evidencias científicas, la desconexión entre científicos y gestores sigue siendo la norma.

\section{¿Qué determina la vulnerabilidad de una especie frente al cambio climático?}

La vulnerabilidad de una especie ante el calentamiento global hace referencia a su capacidad para responder y adaptarse a las nuevas condiciones climáticas, de manera que aquellas especies que tengan una capacidad de respuesta limitada, serán las más vulnerables (McKinney 1997). En este sentido, la vulnerabilidad de una especie ante el cambio climático dependerá fundamentalmente de dos factores: i) su capacidad para mantener poblaciones en su área de distribución actual a pesar del cambio en las condiciones ambientales (persistencia), y ii) el potencial para colonizar zonas 
Cuadro 1. El cambio climático en España:

La región Euromediterránea ha sido identificada como una de las más vulnerables ante el cambio climático puesto que se prevén incrementos significativos en la temperatura media y en la severidad de las sequías, especialmente en la franja meridional (Sala et al. 2000). En el caso de España, las predicciones basadas en escenarios futuros con emisiones medias-altas de gases de efecto invernadero (escenario A2 de SRESIPCC) establecen que durante el periodo 2070-2100 las temperaturas en las zonas del interior peninsular podrían alcanzar valores de $5-8^{\circ} \mathrm{C}$ por encima de los actuales y, en general, se espera una acentuación en el gradiente latitudinal de aridez que ya existe en la Península Ibérica, con la práctica desaparición de las condiciones más húmedas y frías y la llegada de regímenes climáticos similares a los que pueden hoy en día encontrarse en el norte de África (Losada 2011; Fig. 1).

actualmente deshabitadas pero que serán climáticamente favorables en el futuro (migración; Williams et al. 2008; Bellard et al. 2012).

En el primer caso, las especies con mayor capacidad de persistencia serán aquellas que tengan amplios rangos de tolerancia climática y alta plasticidad para adecuar sus preferencias a las nuevas condiciones climáticas en las localidades habitadas. Así, sin necesidad de modificar su rango de distribución, las especies pueden lidiar con el cambio en las condiciones climáticas de sus localidades si estas nuevas condiciones se encuentran dentro de sus rangos de tolerancia. Pese a que los estudios que evalúan la persistencia potencial de los organismos al cambio climático son escasos, la tolerancia a las altas temperaturas así como la capacidad de aclimatación térmica han sido identificadas como buenos indicadores de la viabilidad futura de las poblaciones y/o especies en sus emplazamientos actuales (ej. Deutsch et al. 2008; Somero 2010). Incluso cuando el cambio en las condiciones ambientales supere la tolerancia para alguna de las variables climáticas, las especies pueden resistir el cambio climático modificando su fenología, su ritmo de actividad diaria o sus preferencias de hábitat o microhábitat $\mathrm{y}$, a mayor escala temporal, las poblaciones pueden incluso variar sus frecuencias génicas mediante procesos microevolutivos que promuevan la adaptación a las nuevas condiciones locales (ej. Parmesan 2006; Cleland et al. 2012).

En el segundo caso, la modificación del área de distribución (migración) puede considerarse el mecanismo de respuesta más inmediato; es decir, si el cambio en las condiciones climáticas no favorece el mantenimiento de una especie en un determinado lugar, ésta intentará migrar a lugares con condiciones más adecuadas (ej. Warren et al. 2001; Parmesan 2006; Wilson et al. 2005). En este caso, el éxito de una especie conllevará una modificación en la forma y/o tamaño de su área de distribución actual que dependerá de i) la cantidad y conectividad de las regiones que en el futuro tendrán condiciones climáticas adecuadas (Krosby et al. 2010) y ii) la capacidad de la especie para desplazarse y colonizar estas nuevas áreas habitables (Thomas et al. 2004).

\section{Modelos de distribución y su aplicación a la gestión de especies y espacios ante el cambio climático}

Para las especies ya amenazadas, es decir, aquellas especies que se encuentran en peligro de desaparición debido al efecto de factores de estrés natural o antropogénico (Hernández-Manrique et al. en prensa), el cambio climático puede constituir una nueva e importante amenaza, ya sea actuando de forma aislada o sinérgicamente junto a otras (Brook et al. 2008). Por tanto, es necesario incluir en estos casos medidas específicas en los planes de gestión de estas especies y de los espacios en donde habitan que permitan mitigar el impacto del cambio climático (Lobo et al. 2011).

Hasta la fecha, la mayor parte de los estudios que han evaluado la vulnerabilidad de las especies ante el cambio climático se basan

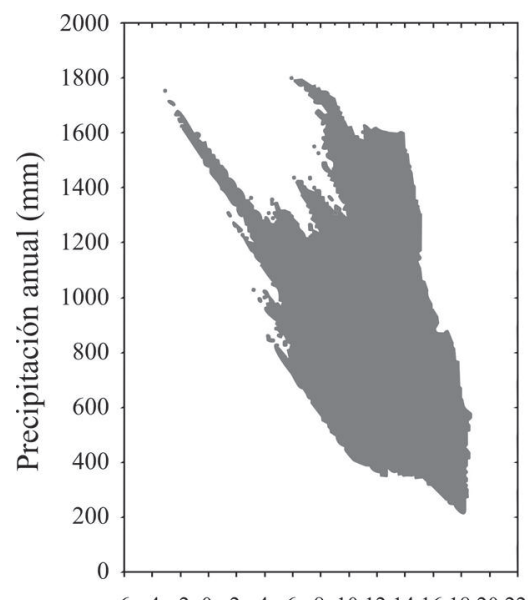

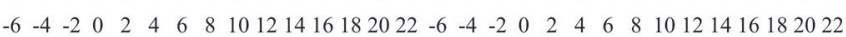
Presente

Temperatura media anual $\left({ }^{\circ} \mathrm{C}\right)$
Figura 1. Valores de temperatura media y precipitación anual de todas las celdas ibéricas de $1 \mathrm{~km}^{2}$ en el presente y en un escenario climático futuro (modelo CCM3 que asume un escenario para el año 2100 donde se duplican las emisiones de efecto invernadero, siendo equivalente al promedio de las familias de escenarios IPCC). Obsérvese la disminución general de las precipitaciones, la pérdida de los climas con temperaturas medias o bajas y altas precipitaciones, así como el incremento generalizado de la aridez en comparación con las condiciones climáticas actuales (polígono rojo). Modificado de Lobo (2011).

en los llamados modelos de distribución de especies. Estos modelos establecen la influencia de las variables climáticas sobre la distribución geográfica de una especie para generar mapas de idoneidad climática. El resultado puede proyectarse sobre escenarios de cambio climático al objeto de estimar el impacto potencial del calentamiento global sobre la distribución de las especies (Guisan y Thuiller 2005; Elith y Leathwick 2009).

Pese a que los modelos de distribución de especies per se son dependientes de un buen número de asunciones e incertidumbres (Jiménez-Valverde et al. 2008) que a su vez se ven incrementadas cuando son proyectados sobre escenarios futuros (Thuiller 2004; Araújo et al. 2005, Heikkinen et al. 2006), estos constituyen uno de los pocos procedimientos de que disponemos para anticipar los efectos del cambio climático sobre la distribución de los organismos. Las predicciones derivadas de los modelos de distribución son así de gran utilidad para adaptar los planes de conservación de las especies a un contexto de cambio climático (ej. Thomas et al. 2004; Thuiller et al. 2005; Thomas et al. 2011; Araújo et al. 2011a), pues permiten no sólo hacer una estima del cambio en el área de distribución con condiciones climáticas adecuadas para cada especie (ganancia o pérdida), sino también segregar las áreas que podrían actuar como refugios climáticamente estables de aquellas otras en las que las poblaciones serían más vulnerables como consecuencia de cambios drásticos en sus condiciones climáticas. De manera similar, estas predicciones permiten identificar regiones no ocupadas en la actualidad por las especies, pero que serían climáticamente favorables en el futuro y que, por tanto, podrían actuar como áreas receptoras para estas especies (ver Cuadro 2 para un ejemplo con los vertebrados amenazados de España). Los modelos de distribución de especies pueden ser especialmente relevantes para la gestión y conservación de aquellos grupos que, como en el caso de los invertebrados, carecen de una información exhaustiva sobre su ecología, demografía y/o historia natural y en los que por tanto, otro tipo de evaluaciones de su vulnerabilidad actual y también futura no son posibles (Samways 2007; Cardoso et al. 2011).

La integración de las estimas individuales de cambio en la distribución de las condiciones climáticas adecuadas para múltiples especies tiene, a su vez, una gran utilidad para adaptar las actuales estrategias de protección de áreas en el marco del cambio climático 
Cuadro 2. El cambio climático y las especies de vertebrados amenazados en España:

Según un reciente estudio (Lobo et al. 2011), las especies de vertebrados ya amenazados (según criterios de la UICN) de la Península Ibérica perderán las condiciones climáticas favorables en un $13 \%$ de la superficie que ocupan actualmente (ej. Fig. 2). La tónica general es que la mayor pérdida ocurriría en las zonas de menor altitud y los valles cercanos al sistema Central, Sierra Morena y Picos de Europa, pero también las serranías de Cádiz en el sur, y en el norte las áreas comprendidas entre los límites del País Vasco y Navarra, sur de Galicia y norte de Zamora. Por otro lado, las regiones climáticamente favorables para los vertebrados amenazados Ibéricos se desplazarían a zonas más elevadas (como era de esperar), pero lo que resulta más preocupante es que buena parte de estas zonas se encuentran ya alteradas por la acción humana. La tendencia general en este caso, es que el tercio septentrional de la Península y la cuenca del Guadalquivir actuarían como áreas con escasa capacidad para alojar las distintas especies de vertebrados amenazados en el futuro. Teniendo en cuenta la alta fragmentación de las zonas con usos del suelo naturales en la Península Ibérica, los cambios estimados en las condiciones climáticas podrían suponer importantes impactos en la futura viabilidad de las especies de vertebrados amenazados, incrementándose todavía más su grado de amenaza actual.

(ej. Hannah et al. 2007; Araújo et al. 2011b). Así, considerando estos potenciales desplazamientos en las distribuciones de las especies, se puede i) evaluar la efectividad de las redes actuales de espacios protegidos en la representación actual y futura de la biodiversidad (Araújo et al. 2004), adaptando así sus planes de gestión, y ii) identificar aquellas regiones naturales actualmente sin protección que podrían jugar un papel relevante en la conservación futura de esa diversidad, aportando un valor añadido para la protección de estas zonas. Esta información resulta de gran utilidad para la elaboración de los planes de gestión a aplicar sobre dichas áreas, ya que permite gestionar el territorio anticipándose a la llegada de determinadas especies (Araújo et al. 2011b; ver Cuadro 3 para detalles del efecto del cambio climático en la efectividad de los Parques Nacionales de España).

Cuadro 3. El cambio climático y los Parques Nacionales en España:

En la actualidad, los Parques Nacionales incluyen superficies climáticamente muy heterogéneas y constituyen una red de áreas relativamente poco transformadas por el hombre, que albergan altos porcentajes de la biodiversidad total de la Península Ibérica (Morillo y Gómez-Campo 2000). Sin embargo, un estudio reciente muestra que el cambio climático podría disminuir drásticamente la representatividad climática de los Parques Nacionales (Lobo 2011), reduciendo su actual capacidad para albergar el espectro de condiciones medioambientales peninsulares y, probablemente, también su capacidad para asegurar la persistencia de los organismos que los habitan. De hecho, menos del $2 \%$ de la superficie de algunos parques como Aigüestortes i Estany de Sant Maurici (Fig. 3), Cabañeros, Monfragüe o Picos de Europa, tendría condiciones climáticas similares a las actuales y sólo la heterogeneidad topográfica de Sierra Nevada permitirá que se mantengan en el futuro dentro de su territorio buena parte de las condiciones climáticas actuales.

En general, las regiones colindantes a los Parques Nacionales podrían presentar en el futuro condiciones similares a las que actualmente tienen éstos y, por tanto, podrían ser receptoras potenciales de gran parte de la biodiversidad que actualmente albergan estos espacios protegidos. El cambio climático puede provocar que cada espacio protegido sea receptor en el futuro de fauna y flora que, actualmente, encuentra condiciones favorables en otros territorios y, a la vez, actúe como "emisor" de otras especies. Debido a ello, es urgente y prioritario realizar un manejo integrado de la red de espacios protegidos y sus áreas adyacentes, adecuando además los planes de gestión de cada uno de ellos a fin de vigilar, facilitar y adaptar su jurisdicción a las nuevas presiones y cambios que se avecinan. La delimitación de nuevas áreas con potencial para actuar como "corredores ecológicos", el manejo racional y sostenible del conjunto del territorio y la elaboración de estrategias de adaptación basadas en evidencias científicas deberían ser la norma de actuación si queremos conservar la biodiversidad Ibérica ante el cambio climático.

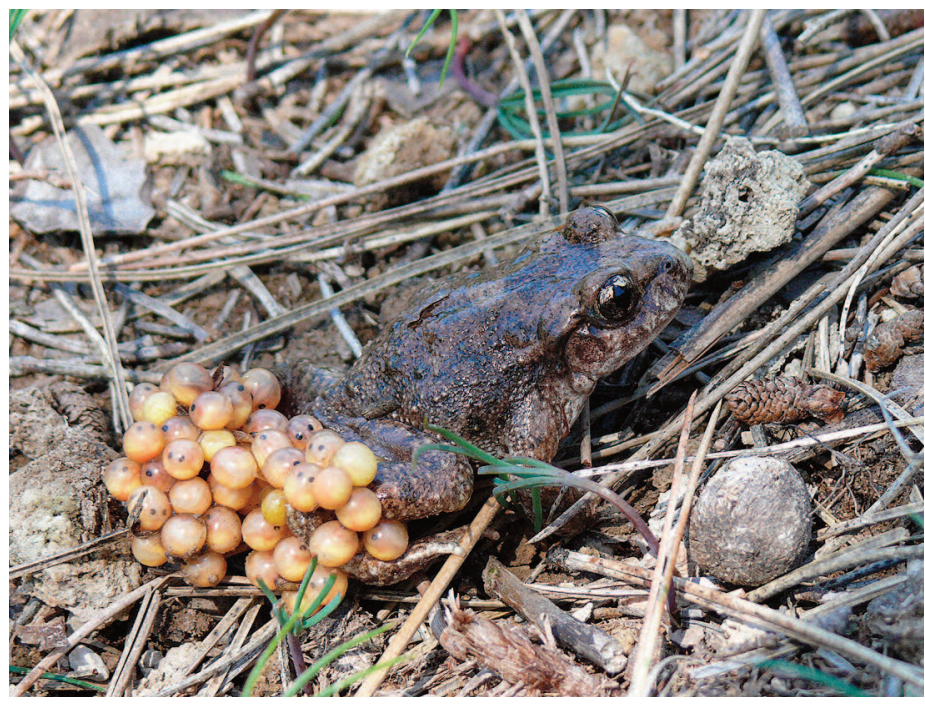

Figura 2. Sapo partero bético (Alytes dickhilleni), endemismo Ibérico catalogado como "Vulnerable" por la IUCN. Los modelos predicen una pérdida sustancial de las condiciones climáticas adecuadas para esta especie en su rango de distribución actual (ej. ver Lobo et al. 2011 y Araújo et al. 2011a). Dicha reducción junto a su limitada capacidad de dispersión podría incrementar significativamente su riesgo de extinción, especialmente en el sur de la Península Ibérica (foto: Jesús Arribas Merino).

\section{Evaluación integrada de la vulnerabilidad de las especies ante el cambio climático y estrategias de conservación}

A pesar de la utilidad de los modelos de distribución, si las evaluaciones de la vulnerabilidad de las especies ante el cambio climático se basan sólo en estimar dónde se encontrarán las condiciones climáticas adecuadas para las mismas en el futuro, se podría subestimar su verdadera capacidad de persistencia en sus emplazamientos actuales. Por otro lado, estas evaluaciones también pueden sobrestimar la capacidad real de las especies para dispersarse y acceder a las nuevas regiones con condiciones futuras adecuadas, ya que aunque las especies tengan mayor espacio con clima adecuado en el futuro, este puede ser inaccesible para aquellas con escasa capacidad de dispersión (Pearson y Dawson 2003; McMahon et al. 2011). Además de las fuentes de incertidumbre mencionadas, las valoraciones de la vulnerabilidad ante el cambio climático basadas exclusivamente en modelos de

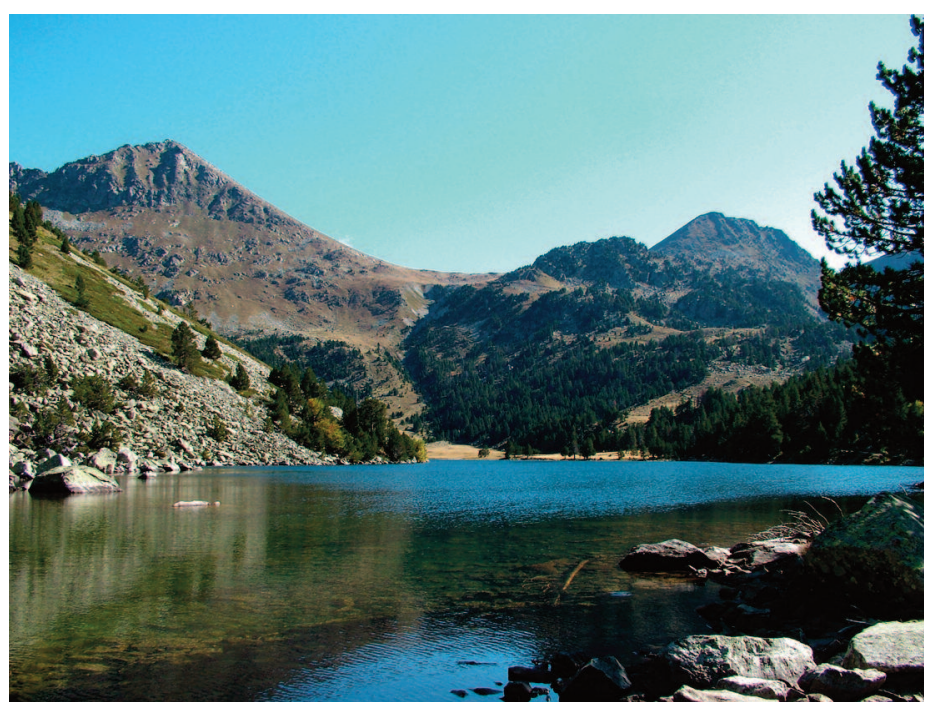

Figura 3. Parque Nacional de Aigüestortes i Estany de Sant Maurici, que verá disminuida su área de representatividad climática en más de un $95 \%$, y por tanto uno de los espacios protegidos donde la incorporación de estrategias para mitigar los efectos de cambio climático en las especies es más urgente (foto: David Sánchez Fernández). 
distribución no suelen proponer acciones precisas que faciliten la adaptación al mismo y, en los casos en los se han propuesto algunas recomendaciones, estas son poco concretas y centradas en la priorización de nuevas áreas a proteger (Heller y Zavaleta 2009).

La evaluación integrada de la vulnerabilidad de las especies al cambio climático, es decir, la combinación de otros determinantes de la vulnerabilidad de las especies (ej. la capacidad de persistencia y de colonización) con las predicciones obtenidas mediante los modelos de distribución, permite evaluar de una manera más precisa el potencial de respuesta de las especies al cambio climático (Williams et al. 2008; Bellard et al. 2012). Estas evaluaciones integradas pueden incorporar de manera secuencial la información de otros determinantes de riesgo a los resultados de modelos de distribución de especies (ver Cuadro 4 para un ejemplo con insectos amenazados de España) o bien, incorporarla de forma intrínseca, a través de la generación de modelos híbridos que acoplan los modelos de distribución con otros que son capaces de simular migración y persistencia (ej. Engler et al. 2009, 2012; Boulangeat et al. 2012). La evaluación integrada de la vulnerabilidad de las especies facilita en gran medida la propuesta de estrategias de gestión concretas ya que permite tener en cuenta la respuesta diferencial de cada especie frente al cambio climático, y por tanto, puede ser especialmente relevante para reevaluar y adaptar los planes de conservación de las especies amenazadas y sus hábitats. Para aquellas especies con elevada capacidad para soportar las condiciones climáticas futuras sin necesidad de dispersarse (ej. por poseer una amplia tolerancia a temperaturas elevadas o capacidad para adaptar sus ciclos de actividad a las nuevas condiciones ambientales), la gestión in situ, esto es, la focalización de los esfuerzos de conservación y mitigación de impactos en las localidades actuales, podría ser la medida más efectiva para promover su viabilidad ante el cambio climático (Arribas et al. 2012). Por otro lado, en el caso de aquellas especies con una escasa capacidad para permanecer en sus localidades actuales, una parte importante de los esfuerzos de
Cuadro 4. Determinantes de la vulnerabilidad de insectos lbéricos amenazados ante el cambio climático y estrategias de conservación:

Un estudio reciente (Arribas et al. 2012) ha evaluado la vulnerabilidad al cambio climático de varias especies amenazadas de coleópteros en la Península lbérica, proponiendo un marco teórico que vincula de manera directa los determinantes de vulnerabilidad de las especies con propuestas de estrategias de gestión concretas para minimizar los efectos del cambio climático (Fig. 4). Las especies pueden verse afectadas por el calentamiento global de manera muy diferente, a pesar de mostrar similares características ecológicas y biogeográficas, y ser incluidas en el mismo nivel de amenaza en base a las categorizaciones tradicionales (ej. IUCN 2001). A modo de ejemplo, este estudio muestra que, aunque las tres especies estudiadas de coleópteros amenazados cohabitan en los ríos salinos Ibéricos (compartiendo gran parte de sus áreas de distribución), el impacto del cambio climático en cada una de ellas podría ser muy dispar, debido a que presentan diferencias importantes en su tolerancia al stress térmico, en su capacidad de dispersión y en la disponibilidad de áreas climáticamente adecuadas en el futuro (ej. Fig. 5).

En este sentido, queda patente que la evaluación de los determinantes de la vulnerabilidad de las especies ante el cambio climático ofrece un complemento efectivo para las herramientas y estrategias de conservación de la biodiversidad existentes, permitiendo focalizar y ajustar los recursos y esfuerzos de gestión para mitigar los impactos del cambio climático sobre las especies y por tanto maximizando su protección, algo que es especialmente relevante para las ya amenazadas.

conservación deberían estar enfocados a facilitar el desplazamiento o modificación de sus áreas de distribución. En este caso, la protección de las áreas que probablemente actuarán en el futuro como refugios es fundamental, así como promover el acceso de las especies a estas nuevas regiones, por ejemplo, mediante la creación de "corredores" biológicos (Hodgson et al. 2009, 2011; Krosby et al. 2010) o, en los casos más extremos, a través de la "colonización asistida" (Hoegh-Guldberg et al. 2008; MoruetaHolme et al. 2010). En este sentido, la evaluación de la capacidad de migración de las especies ofrece una información fundamental
DETERMINANTES de VULNERABILIDAD

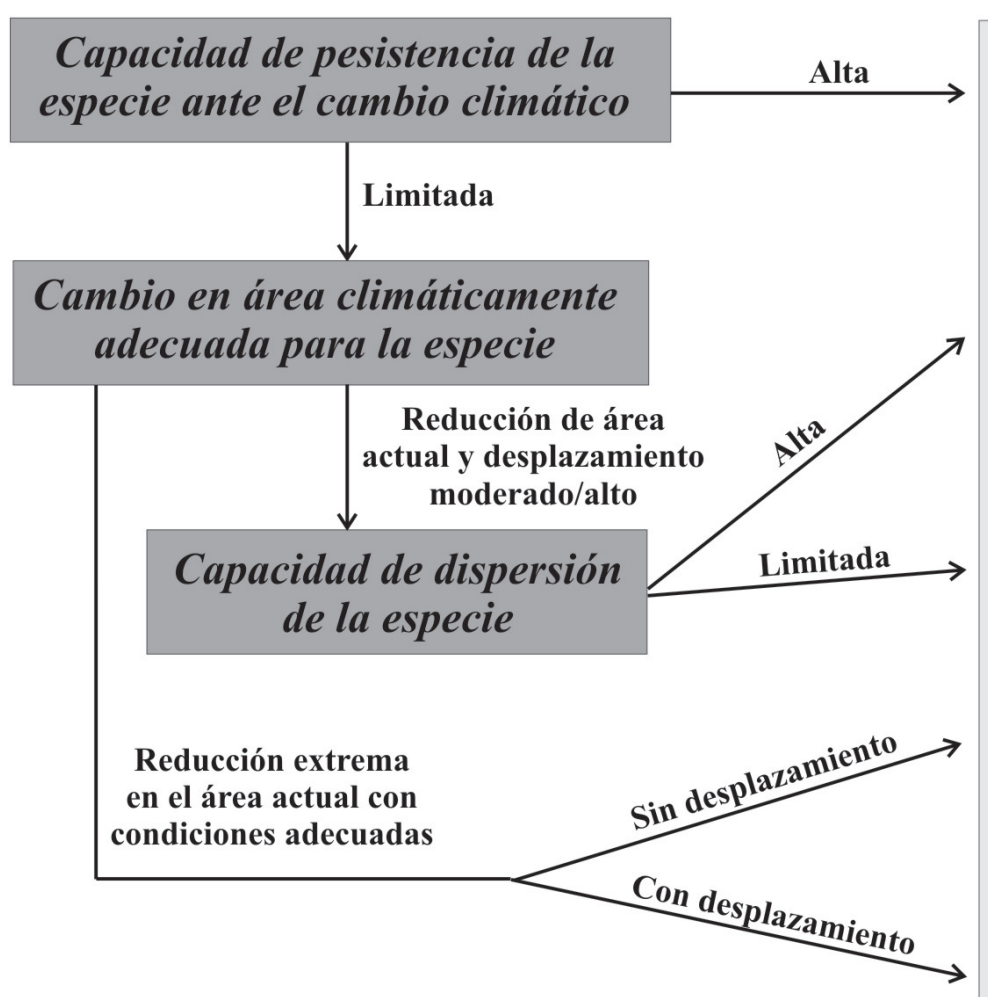

\section{ESTRATEGIAS de CONSERVACIÓN}

Focalización de esfuerzos de conservación en localidades actuales Protección del hábitat y minimización de presiones

Conservación de áreas potencialmente adecuadas en el futuro

Protección del hábitat y minimización de presiones

Incremento en la conectividad de las áreas climáticamente adecuadas Corredores, incremento en la calidad y área de los hábitats Colonización asistida

\section{Medidas intensas para mantener las} poblaciones actuales

Adecuación de hábitats para minimizar los efectos del cambio climático, biomonitorización de poblaciones

Colonización asistida

Biomonitoreo de poblaciones, protección de los habitats y minimización de presiones

Figura 4. Marco teórico para guiar la selección de estrategias de conservación en función de los determinantes de vulnerabilidad de cada especie. Modificado de Arribas et al. (2012). 


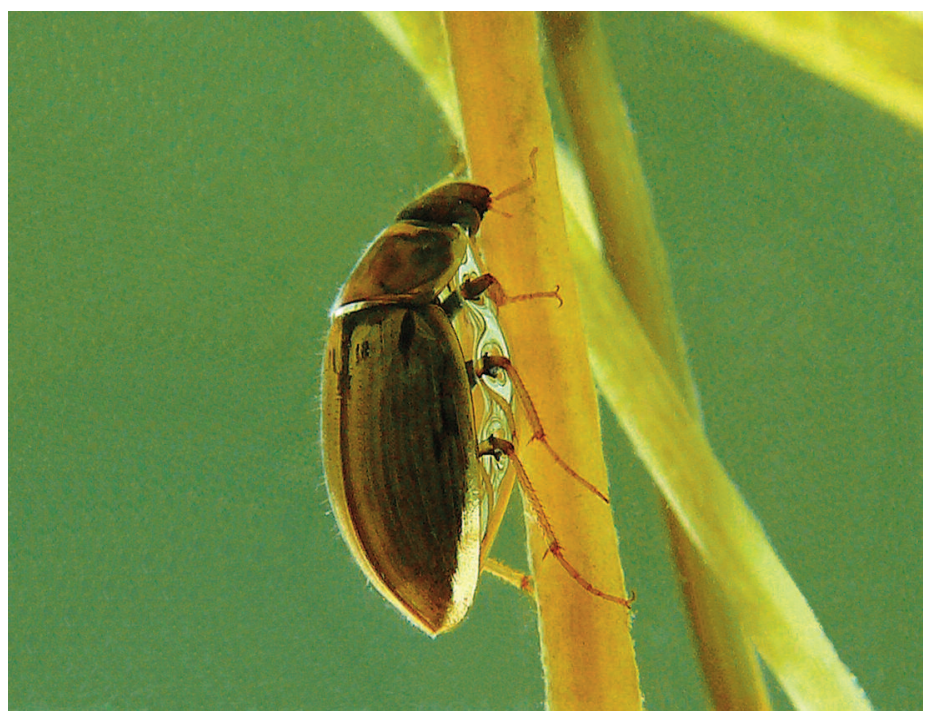

Figura 5. Coleóptero acuático de ríos salinos (Enochrus jesusarribasi IP, ver Arribas et al. en prensa), endemismo Ibérico y propuesto como "Vulnerable" en base a los criterios de la IUCN. Esta especie presenta una capacidad de dispersión muy limitada pero su amplia tolerancia a las temperaturas altas (supervivencia por encima de los $50^{\circ} \mathrm{C}$ ) apunta a una elevada capacidad de persistir en sus localidades del sur de la Península Ibérica en el futuro. La focalización de los esfuerzos de gestión en minimizar y mitigar de impactos adicionales sobre estas poblaciones podría ser la estrategia más coste-efectiva para su conservación (foto: Jesús Arribas Merino)

para determinar las estrategias específicas a aplicar a fin de facilitar la colonización a esas nuevas áreas de las poblaciones más vulnerables.

Existe una desconexión evidente entre científicos y gestores del medio natural; entre conocimiento científico y práctica de conservación. Esta falta de comunicación está especialmente acentuada en el caso de la adaptación de las estrategias de gestión de la biodiversidad ante el cambio climático, en otras palabras, la mayor parte de los esfuerzos de modelado que se hacen en las investigaciones sobre el cambio climático no tienen un impacto real en la forma en la que gestionamos nuestra biodiversidad. Esta situación es debida en parte al importante grado de incertidumbre asociado a los resultados de los modelos de distribución de especies proyectados al futuro, pero también a la escasa atención y participación de expertos en el diseño y puesta en marcha de las estrategias de conservación. En este sentido, las evaluaciones integradas de la vulnerabilidad de las especies ante el cambio climático, por suponer una importante reducción de la incertidumbre asociada a los resultados teóricos, podrían tender un puente fundamental para acercar ambas partes y llevar gestión efectiva de la biodiversidad en el contexto del calentamiento global. Así, para los científicos, la obtención de datos experimentales que permitan estimar la capacidad de persistencia y migración de las especies, junto con el desarrollo de metodologías y protocolos que combinen dicha información con los modelos de distribución y faciliten su traducción a estrategias de gestión concretas, deberían ser objetivos prioritarios a fin de lidiar con el desfase teóricopráctico. Por el lado de los gestores, es necesario un fuerte compromiso y la focalización de esfuerzos y recursos de modo que las evidencias científicas sean consideradas y constituyan un elemento fundamental para la gestión de los sistemas naturales. En el caso de España, a pesar de que dicha conexión es una necesidad oficialmente reconocida (ej. ver referencias: Oficina española de cambio climático 2008, Bladé et al. 2010; Felicísimo et al. 2011), el proceso de adecuación de los planes y estrategias de conservación al cambio climático ha sido escasamente afrontado. Se trata de un reto necesario y urgente en el que todos estamos implicados y del que dependerá el futuro de nuestra biodiversidad.

\section{Agradecimientos}

A Jesús Arribas por las fotografías proporcionadas y a todos los compañeros del grupo de investigación de Ecología Acuática de la Universidad de Murcia y Carmelo Andújar por su contribución en las distintas fases de este estudio. El estudio ha sido financiado por una beca predoctoral (P. Arribas) y una postdoctoral (P. Abellán) ambas del Ministerio de Economía y Competitividad, una beca postdoctoral del mismo Ministerio dentro del programa Juan de la Cierva (D. Sánchez-Fernández) y por el proyecto del Ministerio de Ciencia e Innovación CGL2010-15378 (J. Velasco).

\section{Referencias}

Akcakaya, H.R., Butchart, S.H.M., Mace, G.M., Stuart, S.N., Hilton-Taylor, C. 2006. Use and misuse of the IUCN Red List Criteria in projecting climate change impacts on biodiversity. Global Change Biology 12:20372043.

Araújo, M.B., Cabeza, M., Thuiller, W., Hannah, L., Williams, P.H. 2004. Would climate change drive species out of reserves? An assessment of existing reserve-selection methods. Global Change Biology 10:16181626.

Araújo, M.B., Whittaker, R.J., Ladle, R.J., Erhard, M. 2005. Reducing uncertainty in projections of extinction risk from climate change. Global Ecology and Biogeography 14:529-538.

Araújo, M.B., Guilhaumon, F., Rodrigues-Neto, D., Pozo-Ortego, I., GómezCalmaestra, R. 2011a. Impactos, vulnerabilidad y adaptación al cambio climático de la biodiversidad española. 2. Fauna de vertebrados. Dirección general de medio Natural y Política Forestal. Ministerio de Medio Ambiente, y Medio Rural y Marino. 640 pp. Madrid, España.

Araújo, M.B., Alagador, D., Cabeza, M., Nogués-Bravo, D., Thuiller, W. 2011b. Climate change threatens European conservation areas. Ecology Letters 14:484-492.

Arribas, P., Abellán, P., Velasco, J., Bilton, D.T., Millán, A., Sánchez-Fernández, D. 2012. Evaluating drivers of vulnerability to climate change: a guide for insect conservation strategies. Global Change Biology 18:2135-2146

Arribas, P., Andújar, C., Sánchez-Fernández, D., Abellán, P., Millán, A. en prensa. Integrative taxonomy and conservation of cryptic beetles in the Mediterranean region (Hydrophilidae). Zoologica Scripta. DOI: 10.1111/zsc. 12000.

Bellard, C., Bertelsmeier, C., Leadley, P., Thuiller, W., Courchamp, F. 2012. Impacts of climate change on the future of biodiversity. Ecology Letters 15:365-377.

Bladé, I., Cacho, I., Castro-Díez, Y., Gomís, D., González-Sampériz, P., Miguez-Macho, G., et al. 2010. Climate in Spain: past, present and future. Regional climate change assessment report. Red telemática CLIVAREspaña, Ministerio de Medio Ambiente y Medio Rural y Marino. 83 pp. Madrid, España.

Boulangeat, I., Gravel, D., Thuiller, W. 2012 Accounting for dispersal and biotic interactions to disentangle the drivers of species distributions and their abundances. Ecology Letters 15:584-593

Brook, B.W., Sodhi, N.S., Bradshaw, C.J.A. 2008. Synergies among extinction drivers under global change. Trends in Ecology and Evolution 23:453-460.

Cardoso, P., Erwin, T.L., Borges, P.A.V., New, T.R. 2011. The seven impediments in invertebrate conservation and how to overcome them. Biological Conservation 144:2647-2655.

Cleland, E.E., Allen, J.M., Crimmins, T.M., Dunne, J.A., Pau, S., Travers, S.E., Zavaleta, E.S., Wolkovich, E.M. 2012. Phenological tracking enables positive species responses to climate change, Ecology 93:17651771

Deutsch, C.A., Tewksbury, J.J., Huey, R.B., Sheldon, K.S., Ghalambor, C.K., Haak, D.C., Martin, P.R. 2008. Impacts of climate warming on terrestrial ectotherms across latitude. Proceedings of the National Academy of Sciences of the United States of America 105:6668-6672.

Elith, J., Leathwick, J.R. 2009. Species distribution models: ecological explanation and prediction across space and time. Annual Review of Ecology Evolution and Systematics 40:677-697.

Engler, R., Randin, C.F., Vittoz, P., Czáka, T., Beniston, M., Zimmermann, N.E., Guisan, A. 2009 Predicting future distributions of mountain plants under climate change: Does dispersal capacity matter? Ecography 32:34-45.

Engler, R., Hordijk, W., Guisan, A. 2012. The MIGCLIM R package - seamless integration of dispersal constraints into projections of species distribution models. Ecography 35:872-878. 
Felicísimo, Á.M., Muñoz, J., Villalba, C.J., Mateo, R.G. 2011. Impactos, vulnerabilidad y adaptación al cambio climático de la biodiversidad española. 2. Flora y vegetación. Oficina Española de Cambio Climático, Ministerio de Medio Ambiente y Medio Rural y Marino. 552 pp. Madrid, España.

Guisan, A., Thuiller, W. 2005. Predicting species distribution: offering more than simple habitat models. Ecology Letters 8:993-1009.

Hannah, L., Midgley, G.F., Andelmand, S., Araújo, M.B., Hughes, G., Martinez-Meyer, E., Pearson, R., Williams, P.H. 2007. Protected area needs in a changing climate. Frontiers in Ecology and Environment 5:131-138.

Heikkinen, R.K., Luoto, M., Araújo, M.B., Virkkala, R., Thuiller, W., Sykes, M.T. 2006 Methods and uncertainties in bioclimatic envelope modelling under climate change. Progress in Physical Geography 30:751-777.

Heller, N.E., Zavaleta, E.S. 2009. Biodiversity management in the face of climate change: a review of 22 years of recommendations. Biological Conservation 142:14-32.

Hernández-Manrique, O.L., Sánchez-Férnandez, D., Numa, C., Galante, E., Verdú, J.R., Lobo, J.M. en prensa Extinction trends of threatened invertebrates in peninsular Spain. Journal of Insect Conservation. Doi.: 10.1007/s10841-012-9502-3

Hodgson, J.A., Moilanen, A., Wintle, B.A., Thomas, C.D. 2011. Habitat area, quality and connectivity: striking the balance for efficient conservation. Journal of Applied Ecology 48:148-152.

Hodgson, J.A., Thomas, C.D., Wintle, B.A., Moilanen, A. 2009. Climate change, connectivity and conservation decision making: back to basics. Journal of Applied Ecology 46:964-969.

Hoegh-Guldberg, O., Hughes, L., Mclntyre, S., Lindenmayer, D.B., Parmesan, C., Possingham, H.P., Thomas, C.D. 2008. Assisted colonization and rapid climate change. Science 321:345-346.

IUCN 2001. Red List categories and criteria: version 3. 1. Gland and Cambridge, UK

Jiménez-Valverde, A., Lobo, J.M., Hortal, J. 2008. Not as good as they seem: the importance of concepts in species distribution modelling. Diversity and Distributions 14:885-890.

Kareiva, P.C. (Coord.) 2008. Synthesis and conclusions. En: Julius, S.H., West, J.M., (eds.). Preliminary Review of Adaptation Options for Climate-Sensitive Ecosystems and Resources, pp. 622-689. Environmental Protection Agency. Washington DC, USA.

Krosby, M., Tewksbury, J., Haddad, N.M., Hoekstra, J. 2010. Ecological connectivity for a changing climate. Conservation Biology 24:1686-1689.

Lobo, J.M. 2011. Vulnerabilidad de las áreas protegidas y de zonas de interés para la biodiversidad ante el cambio climático. En: Álvarez-Uría, P., De la Cruz, J.L. (Coords.). Biodiversidad en España. Base de la Sostenibilidad ante el Cambio Global, pp. 360-368. Observatorio de la Sostenibilidad de España, Ministerio de Medio Ambiente y Medio Rural y Marino. Madrid, España.

Lobo, J.M., Aragón, P., Sánchez-Fernández, D. 2011. Las Especies. En: Álvarez-Uría, P., De la Cruz, J.L. (Coords.). Biodiversidad en España. Base de la Sostenibilidad ante el Cambio Global, pp. 323-333. Observatorio de la Sostenibilidad de España, Ministerio de Medio Ambiente y Medio Rural y Marino. Madrid, España.

Losada, I. 2011. Efecto del cambio climático. En: En: Álvarez-Uría, P., De la Cruz, J.L. (Coords.). Biodiversidad en España. Base de la Sostenibilidad ante el Cambio Global, pp. 175-195. Observatorio de la Sostenibilidad de España, Ministerio de Medio Ambiente y Medio Rural y Marino. Madrid, España.

McKinney, M.L. 1997. Extinction vulnerability and selectivity: combining ecological and paleontological views. Annual Review of Ecology and Systematics 28:495-516.
McMahon, S.M., S.P. Harrison, W.S. Armbruster, P.J. Bartlein, C.M. Beale, M.E. Edwards, J. Kattge, G. Midgley, X. Morin, I.C. Prentice, 2011, Improving assessment and modelling of climate change impacts on global terrestrial biodiversity. Trends in Ecology and Evolution 26:249-259.

Morillo, C., Gómez-Campo, C. 2000. Conservation in Spain, 1980-2000. Biological Conservation 95:165-174.

Morueta-Holme, N., Flojgaard, C., Svenning, J.C. 2010. Climate cange risks and conservation implications for a threatened small-range mammal. PLoS One 5:e10360.

Ohlemuller, R., Gritti, E.S., Sykes, M.T., Thomas, C.D. 2006. Quantifying components of risk for European woody species under climate change. Global Change Biology 12:1788-1799.

Parmesan, C. 2006. Ecological and evolutionary responses to recent climate change. Annual Review of Ecology Evolution and Systematics 37:637-669.

Pearson, R.G., Dawson, T.P. 2003. Predicting the impacts of climate change on the distribution of species: are bioclimate envelope models useful? Global Ecology and Biogeography 12:361-371.

Oficina española de cambio climático 2008. Plan nacional de adaptación al cambio climático. Marco para la coordinación entre Administraciones Públicas para las actividades de evaluación de impactos, vulnerabilidad y adaptación al cambio climático. Ministerio de Medio Ambiente y Medio Rural y Marino. 59 pp. Madrid, España.

Sala, O.E., Chapin, F.S., Armesto J.J., Berlow, E., Bloomfield, J., Dirzo, R., et al. 2000. Biodiversity - global biodiversity scenarios for the year 2100 . Science 287:1770-1774.

Samways, M.J. 2007. Insect conservation: a synthetic management approach. Annual Review of Entomology 52:465-487.

Somero, G.N. 2010. The physiology of climate change: how potentials for acclimatization and genetic adaptation will determine 'winners' and 'losers'. Journal of Experimental Biology 213:912-920.

Thomas, C.D., Cameron, A., Green, RE., Bakkenes, M., Beaumont, L.J., Collingham, Y.C., et al. 2004. Extinction risk from climate change. Nature 427:145-148.

Thomas, C.D., Hill, J.K., Anderson, B.J., Bailey, S., Beale, C.M., Bradbury, R.B., et al. 2011. A framework for assessing threats and benefits to species responding to climate change. Methods in Ecology and Evolution 2:125-142.

Thuiller, W. 2004. Patterns and uncertainties of species'range shifts under climate change. Global Change Biology 10:2020-2027.

Thuiller, W., Lavorel, S., Araújo, M.B., Sykes, M.T., Prentice, I.C. 2005. Climate change threats to plant diversity in Europe. Proceedings of the $\mathrm{Na}$ tional Academy of Sciences of the United States of America 102:8245-8250.

Thuiller, W., Lavergne, S., Roquet, C., Boulangeat, I., Araújo, M.B. 2011. Consequences of climate change on the Tree of Life in Europe. Nature 448:550-552.

Warren, M.S., Hill, J.K., Thomas, J.A., Asher, J., Fox, R., Huntley, B., Roy, D.B., Telfer, M.G., Jeffcoate, S., Harding, P., Jeffcoate, G., Willis, S.G., Greatorex-Davies, J.N., Moss, D., Thomas, C.D. 2001. Rapid responses of British butterflies to opposing forces of climate and habitat change. Nature 414:65-69.

Williams, S.E., Shoo, L.P., Isaac, J.L., Hoffmann, A.A., Langham, G. 2008. Towards an integrated framework for assessing the vulnerability of species to climate change. Plos Biology 6:2621-2626.

Wilson, R.J., Gutiérrez, D., Gutiérrez, J., Martínez, D., Agudo, R., Monserrat, V.J. 2005. Changes to the elevational limits and extent of species ranges associated with climate change. Ecology Letters 8:1138-1146. 